\title{
Dusty phenomena in the vicinity of giant exoplanets
}

\author{
O. V. Arkhypov ${ }^{1}$, M. L. Khodachenko ${ }^{1,2}$, and A. Hanslmeier ${ }^{3}$ \\ ${ }^{1}$ Space Research Institute (IWF), Austrian Academy of Sciences, Schmiedlstrasse 6, 8042 Graz, Austria \\ e-mail: oleksiy.arkhypov@oeaw.ac.at \\ ${ }^{2}$ Institute of Laser Physics, SB RAS, Novosibirsk 630090, Russia \\ e-mail: maxim. khodachenko@oeaw. ac . at \\ ${ }^{3}$ Institute of Physics, Karl-Franzens University of Graz, Universitätsplatz 5, 8010 Graz, Austria \\ e-mail: arnold.hanslmeier@uni-graz.at
}

Received 19 August 2019 / Accepted 1 October 2019

\begin{abstract}
Context. Hitherto, searches for exoplanetary dust have focused on the tails of decaying rocky or approaching icy bodies only at short circumstellar distances. At the same time, dust has been detected in the upper atmospheric layers of hot jupiters, which are subject to intensive mass loss. The erosion and/or tidal decay of hypothetic moonlets might be another possible source of dust around giant gaseous exoplanets. Moreover, volcanic activity and exozodiacal dust background may additionally contribute to exoplanetary dusty environments.

Aims. In the present study, we look for photometric manifestations of dust around different kinds of exoplanets (mainly giants). Methods. We used linear approximation of pre- and post-transit parts of the long-cadence transit light curves (TLCs) of 118 Kepler objects of interest after their preliminary whitening and phase-folding. We then determined the corresponding flux gradients $G_{1}$ and $G_{2}$, respectively. These gradients were defined before and after the transit border for two different time intervals: (a) from 0.03 to 0.16 days and (b) from 0.01 to 0.05 days, which correspond to the distant and adjoining regions near the transiting object, respectively. Statistical analysis of gradients $G_{1}$ and $G_{2}$ was used for detection of possible dust manifestation.

Results. It was found that gradients $G_{1}$ and $G_{2}$ in the distant region are clustered around zero, demonstrating the absence of artifacts generated during the light curve processing. However, in the adjoining region, 17 cases of hot jupiters show significantly negative gradients, $G_{1}$, whereas the corresponding values of $G_{2}$ remain around zero. The analysis of individual TLCs reveals the localized pretransit decrease of flux, which systematically decreases $G_{1}$. This effect was reproduced with the models using a stochastic obscuring precursor ahead of the planet.

Conclusions. Since only a few TLCs show the presence of such pre-transit anomalies with no analogous systematic effect in the post-transit phase, we conclude that the detected pre-transit obscuration is a real planet-related phenomenon. Such phenomena may be caused by dusty atmospheric outflows or background circumstellar dust compressed in front of the mass-losing exoplanet, the study of which requires dedicated physical modeling and numeric simulations. Of certain importance may be the retarding of exozodiacal dust relative to the planet by the Poynting-Robertson effect leading to dust accumulation in electrostatic or magnetic traps in front of the planet.
\end{abstract}

Key words. planets and satellites: general - interplanetary medium - zodiacal dust

\section{Introduction}

Exoplanetary environments are nowadays the focus of extensive theoretical modeling and dedicated observations, traditionally in spectral lines (Lammer \& Khodachenko 2015). The available broadband photometry may provide additional valuable environmental information for significantly larger number of objects, as compared to those studied by spectral methods. At the same time, only the signatures of the dust component are detectable in such studies. Until now, the manifestations of dust have been noticed on decaying rocky planets in the form of tails (e.g., Brogi et al. 2012; Budaj 2013; Garai 2018; Sanchis-Ojeda et al. 2015). The presence of dust was also reported at altitudes of $\sim 3000 \mathrm{~km}$ in gaseous giant exoplanets (Huitson et al. 2012; Wang \& Dai 2019), and it is quite possible that such dust is dragged by the escaping planetary upper atmospheric material driven by stellar X-ray and ultraviolet (XUV) heating and tidal forces (Shaikhislamov et al. 2016). Among the sources of the dust at giant exoplanets could be the erosion of moonlets by meteoroids and plasma flows, tidal decay and evaporation of heated and liquified satellites, satellite volcanic activity, and magnetospheric capture of interplanetary (e.g., exozodiacal) dust (Spahn et al. 2019). The dust product of any of these processes can manifest itself as dusty obscuring matter (DOM), which transits over a stellar disk ahead of or behind the planet, generating tiny anomalies in the exoplanetary transit light curves (TLCs), especially before and after planetary eclipses.

In that respect, the TLCs provided by the space telescope Kepler represent promising but still superficially studied source of information on the pre/post-transit anomalies and the related manifestations of DOM. Hitherto, the out-of-transit parts of exoplanetary TLCs have only been studied for a cumulative search of exomoons and evaluation of particular candidates around Kepler1625b (Teachey et al. 2018). Here we analyze for the first time the transit vicinities of many individual Kepler objects of interest (KOIs). The maximal duration of available data records and their highest precision make Kepler data the best choice to search for tenuous photometric effects of DOM.

The analysis presented here involves a specially elaborated method, which is explained in Sect. 2. The obtained results are described in Sect. 3 and conclusions are presented in Sect. 4. 

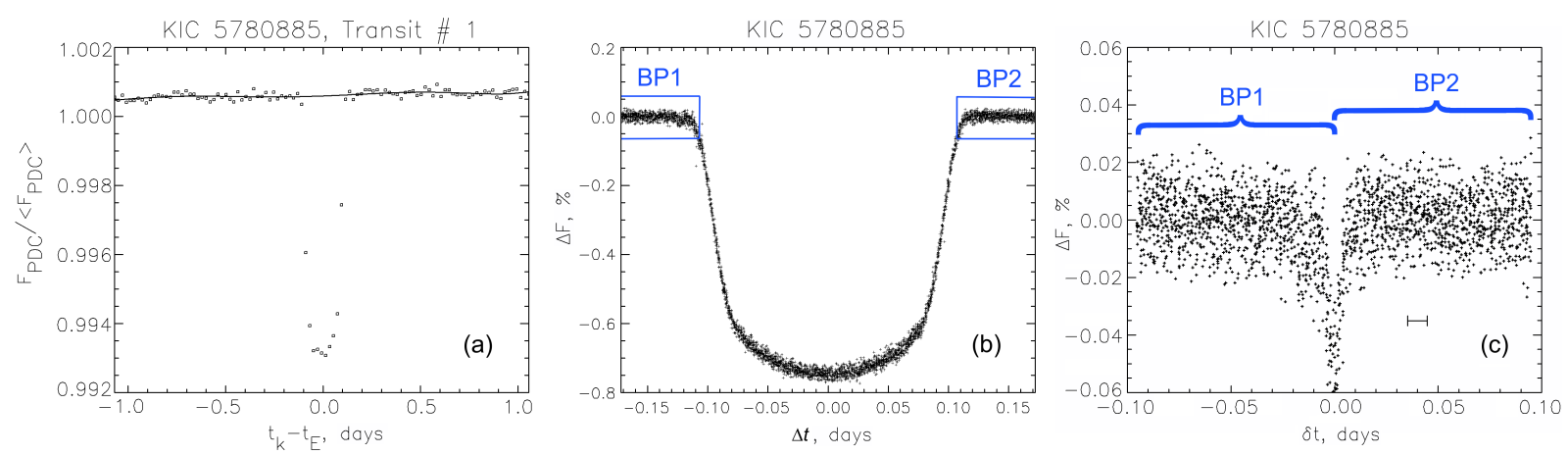

Fig. 1. Example of the light curve processing for the star KIC 5780885. Panel a: approximation of the transit background (open squares) with a sixth-order polynomial $F_{b}\left(t_{k}\right)$ (solid line); panel $b$ : phase-folded TLC, $\Delta F(\Delta t)$, with near-transit border parts BP1 and BP2 indicated (blue boxes); panel $c$ : clipped border parts (labeled) vs. the border distance $\delta t$ ready for comparison. The black horizontal bar in $(c)$ corresponds to the radius ingress (egress) time of the planet.
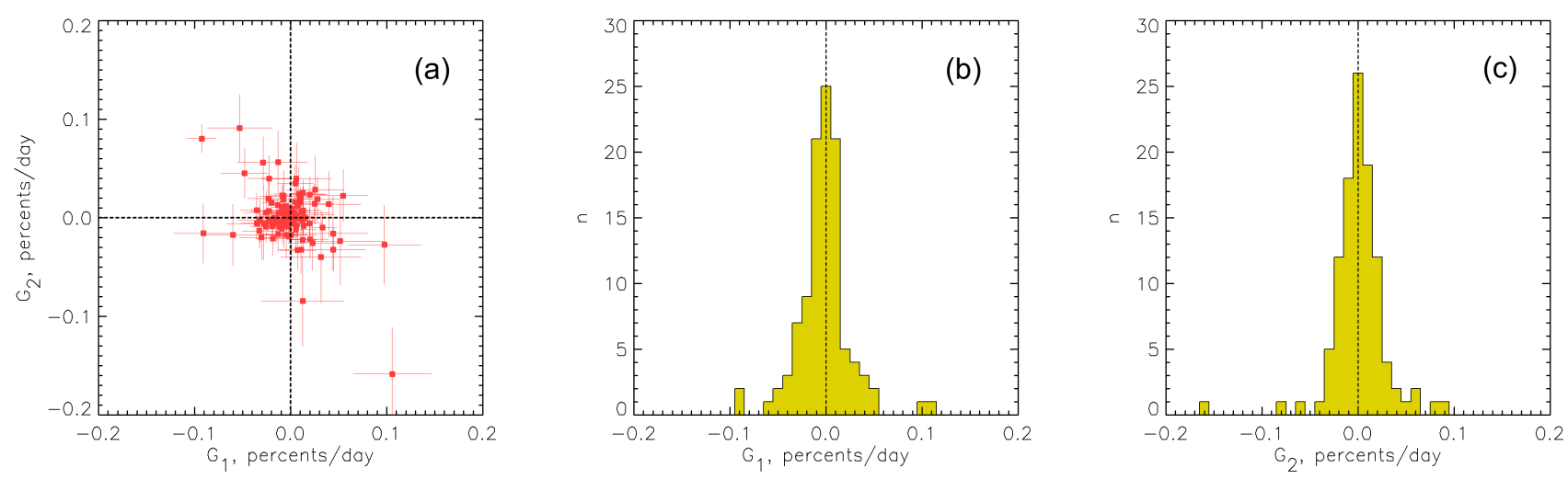

Fig. 2. Distributions of the pre-ingress, $G_{1}$, and post-egress, $G_{2}$, gradient estimates of TLC for the distant pre- and post-transit regions with $\tau_{\min }=0.03$ days and $\tau_{\max }=0.16$ days for 114 KOIs with errors $<0.05 \% /$ day: panel a: $G_{2}$ vs. $G_{1}$ diagram; panel b: histogram of $G_{1}$; panel $c$ : histogram of $G_{2}$. The value $n$ is the number of estimates within a bin of histogram.

\section{Analysis method and stellar set}

A natural way to detect DOM structures co-moving with the transiting object in broadband photometry is to search for flux variations a short time before the ingress of the planet on the stellar disk and after the egress. For this purpose we use the publicly available light curves from the Kepler mission (NASA Exoplanet Archive $\left.^{1}\right)$ after Pre-search Data Conditioning ( $F_{\text {PDC }}$ hereinafter), where instrumental drifts, focus changes, and thermal transients are removed or suppressed (Jenkins et al. 2010). Our survey uses the long-cadence data with photon accumulation (i.e., exposure) period of $\delta t_{L}=29.4 \mathrm{~min}$. This approach provides the highest precision of the input light curves, although it cannot resolve the details of the ingress and egress parts of the TLCs. The shortcadence data with the counting period of $\delta t_{S}=1 \mathrm{~min}$, in spite of higher time resolution, are available for a lesser number of stars and not for all quarters; and they also increase the detection threshold of DOM by a factor of $\sqrt{\delta t_{L} / \delta t_{S}}=5.4$.

To remove the residual instrumental drifts as well as the stellar variability at timescales longer than the transit duration, after removal of the transit parts of duration $\Delta t_{\text {tr }}$ plus the margins of \pm 0.01 days, we approximate the normalized light curve $F_{\text {PDC }}\left(t_{k}\right) /\left\langle F_{\text {PDC }}\left(t_{k}\right)\right\rangle$, which covers the time interval $\pm 10 \Delta t_{\text {tr }}$ centered at the particular transit, (see Fig. 1a), with a sixth-order polynomial $F_{b}\left(t_{k}\right)=a t_{k}^{6}+b t_{k}^{5}+c t_{k}^{4}+d t_{k}^{3}+e t_{k}^{2}+f t_{k}+g$, where $t_{k}$ is the flux measurement time, $a, b, c, d, e, f, g$ are the fitted coefficients, and $\left\langle F_{\mathrm{PDC}}\left(t_{k}\right)\right\rangle$ is the average flux of the light curve. This approximation is an iterative process (10 iterations),

\footnotetext{
1 https://exoplanetarchive.ipac.caltech.edu/
}

with the consequent exclusion of remaining stellar flares and transit counts above the threshold $\mid\left[F_{\mathrm{PDC}}\left(t_{k}\right) /\left\langle F_{\mathrm{PDC}}\left(t_{k}\right)\right\rangle\right]-$ $F_{b}\left(t_{k}\right) \mid>3 \sigma_{b}$, where $\sigma_{b}$ is a standard deviation from the approximation $F_{b}\left(t_{k}\right)$. Since the final approximation $F_{b}\left(t_{k}\right)$ is insensitive to the transit (Fig. 1a), we use it as reference level to determine the flux decrease during the transit: $\Delta F_{k}=$ $\left[F_{\mathrm{PDC}}\left(t_{k}\right) /\left\langle F_{\mathrm{PDC}}\left(t_{k}\right)\right\rangle\right]-F_{b}\left(t_{k}\right)$, which is used in further analysis.

This light curve "whitening" procedure enables us to finally obtain a phase-folded TLC $\Delta F(\Delta t)$ (Fig. 1b), where $\Delta t=t_{k}-t_{E}$ is the time counted for each transit with number $E=0,1,2, \ldots$ relative to its mid-time, $t_{E}=t_{0}+P_{\mathrm{tr}} E$. The cumulative reference time $t_{0}$ and the transit period $P_{\text {tr }}$ are taken from the NASA Exoplanet Archive.

To detect the possible manifestation of DOM, which is supposedly concentrated around an exoplanet, the linear gradients $G_{1,2} \equiv \partial(\Delta F) / \partial(\delta t)$ were calculated in the time intervals $-\tau_{\max }<$ $\delta t<-\tau_{\min }$ and $\tau_{\min }<\delta t<\tau_{\max }$ for the pre-ingress and postegress parts of the TLCs, respectively. Here, $\delta t=\Delta t \pm 0.5 \Delta t_{\text {tr }}$ is the border distance time counted from the transit border in folded TLC, calculated with the cumulative transit duration $\Delta t_{\text {tr }}$ from the NASA Exoplanet Archive. The gradients $G_{1}$ and $G_{2}$ were found in two interval ranges: (a) in the adjoining pre/post-transit region, i.e., between $\tau_{\min }=0.01$ days (the half-exposure of the flux record) and $\tau_{\max }=0.05$ days; (b) in the distant region, that is, between $\tau_{\min }=0.03$ days and $\tau_{\max }=0.16$ days, which correspond approximately to the planetocentric distances from $\sim 2$ to $\sim 17$ planetary radii.

Altogether, a set of 118 KOIs with "confirmed" or "candidate" status in the NASA Exoplanet Archive was compiled. 

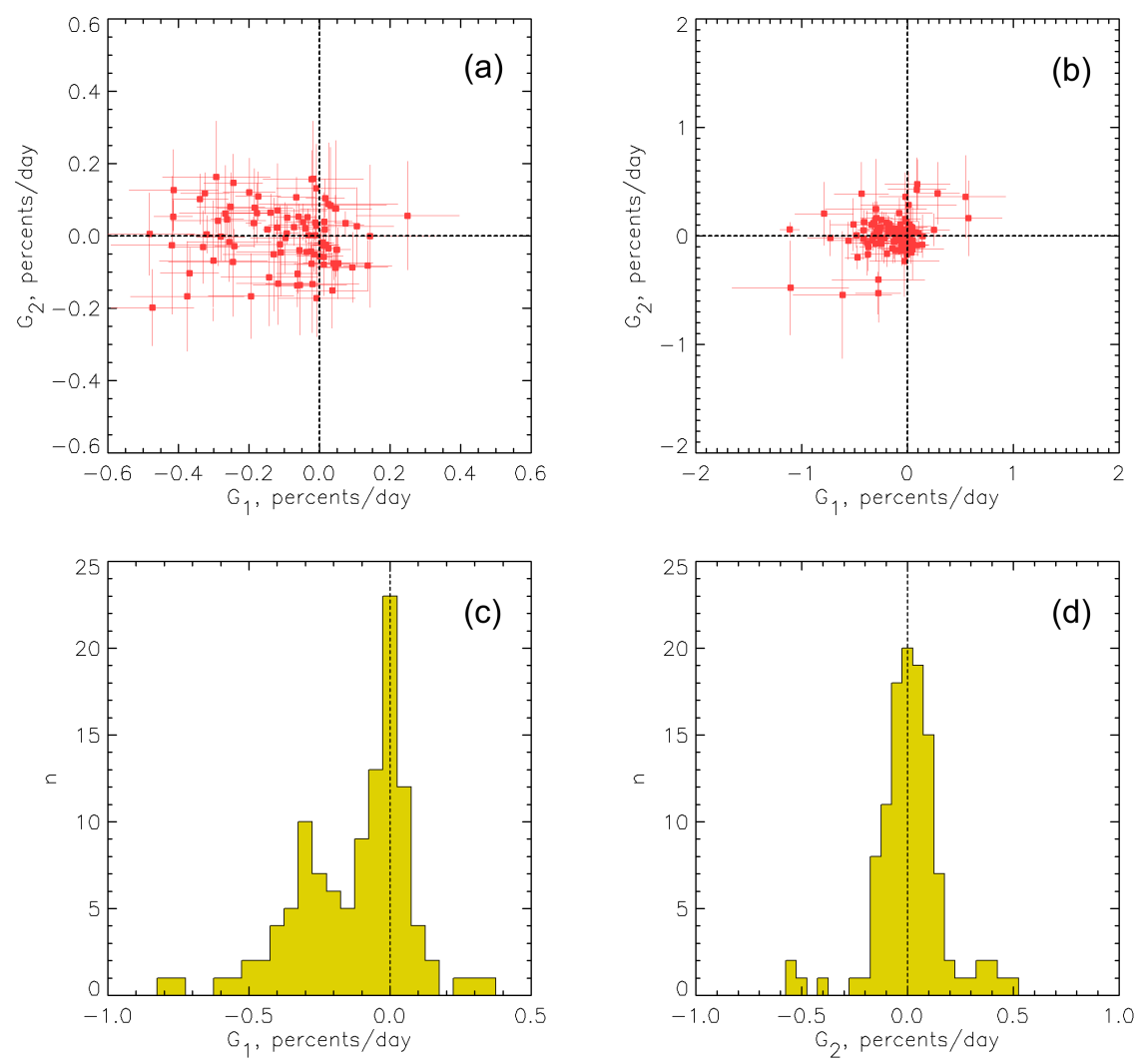

Fig. 3. Distributions of pre-ingress $\left(G_{1}\right)$ and postegress $\left(G_{2}\right)$ gradients estimated in the adjoining regions $\left(\tau_{\min }=0.01\right.$ and $\tau_{\max }=0.05$ days). Panel $a$ : $G_{2}$ vs. $G_{1}$ diagram for $90 \mathrm{KOIs}$ with gradient errors (marked by bars) of less than $0.2 \% /$ day; panel $b$ : diagram analogous to $(a)$, but for all 114 KOIs considered; panel $c$ : histogram of $G_{1}$ for all estimates (i.e., all objects); panel $d$ : histogram of $G_{2}$ for all estimates (i.e., all objects). The value $n$ is the number of estimates within a bin of a histogram.
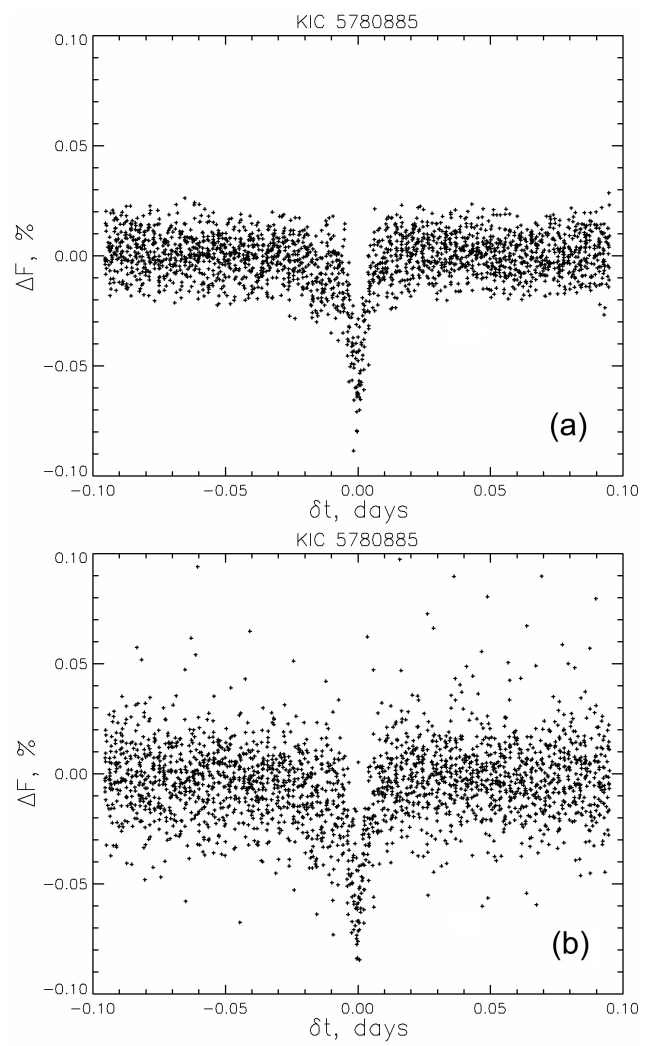

Fig. 4. Effect of imperfect approximation of the transit reference level $F_{b}\left(t_{k}\right)$ in the case of KIC 5780885 in Fig. 1c. Panel $a$ : approximation with a sixth-order polynomial, commonly used in our study, which gives $G_{1}=-0.26 \pm 0.05$ and $G_{2}=0.05 \pm 0.05 \% /$ day; panel $b$ : trial approximation with first-order polynomial covering the whole $P_{\mathrm{tr}}$, which gives $G_{1}=-0.32 \pm 0.10$ and $G_{2}=0.04 \pm 0.11 \% /$ day.
This set consists of selected objects from the lists in Aizawa et al. (2018), whose short-cadence light curves were used to search for exo-rings (i.e., specific structures of the dusty matter around planets). Since in our study we deal with the longcadence data only, the set of corresponding long-cadence light curves of the objects from Aizawa et al. (2018) was extended with other high-quality light curves of objects from the NASA Exoplanet Archive with the nominated signal-to-noise ratio $(S / N) \gtrsim 10^{3}$ (i.e., the transit depth normalized by the mean uncertainty in the flux during the transits as it was published in the KOI cumulative table of the NASA Exoplanet Archive). For the selection of KOIs from the list in Aizawa et al. (2018), the same version of the $\mathrm{S} / \mathrm{N}$ definition as that used in the NASA Exoplanet Archive was considered, but with a less conservative selection threshold of $\gtrsim 100$. In cases of multi-planet systems, the objects with maximal transit depth were taken (one per system). The total set of KOIs selected for our analysis is listed in Table C.1.

To visualize the possible DOM effects, the phase-folded TLC (Fig. 1b) is clipped according to Fig. 1c. The clipping consists in the combination of the near-transit border parts (BP1 and BP2 in Fig. 1b) of the folded TLC to simplify their comparison. The null-punkt of the abscissa scale in Fig. 1c $\delta t=0$ corresponds to the time $\Delta t \pm 0.5 \Delta t_{\mathrm{tr}}$ of the borders of transit. In fact, the observable transit borders in long-cadence light curves are shifted from those, defined as above, on half exposure period, i.e., on $\delta t_{L} / 2$, that is -0.01 and +0.01 days for the transit start- and end- times, respectively. Correspondingly, the clipped border parts of folded TLC show the sharp minimum in Fig. 1c.

\section{Results}

First, we present the results obtained for the distant region with $\tau_{\min }=0.03$ days and $\tau_{\max }=0.16$ days (Fig. 2). One can see that most of the $G_{1,2}$ estimate points cluster around $G_{1}=G_{2}=0$. 

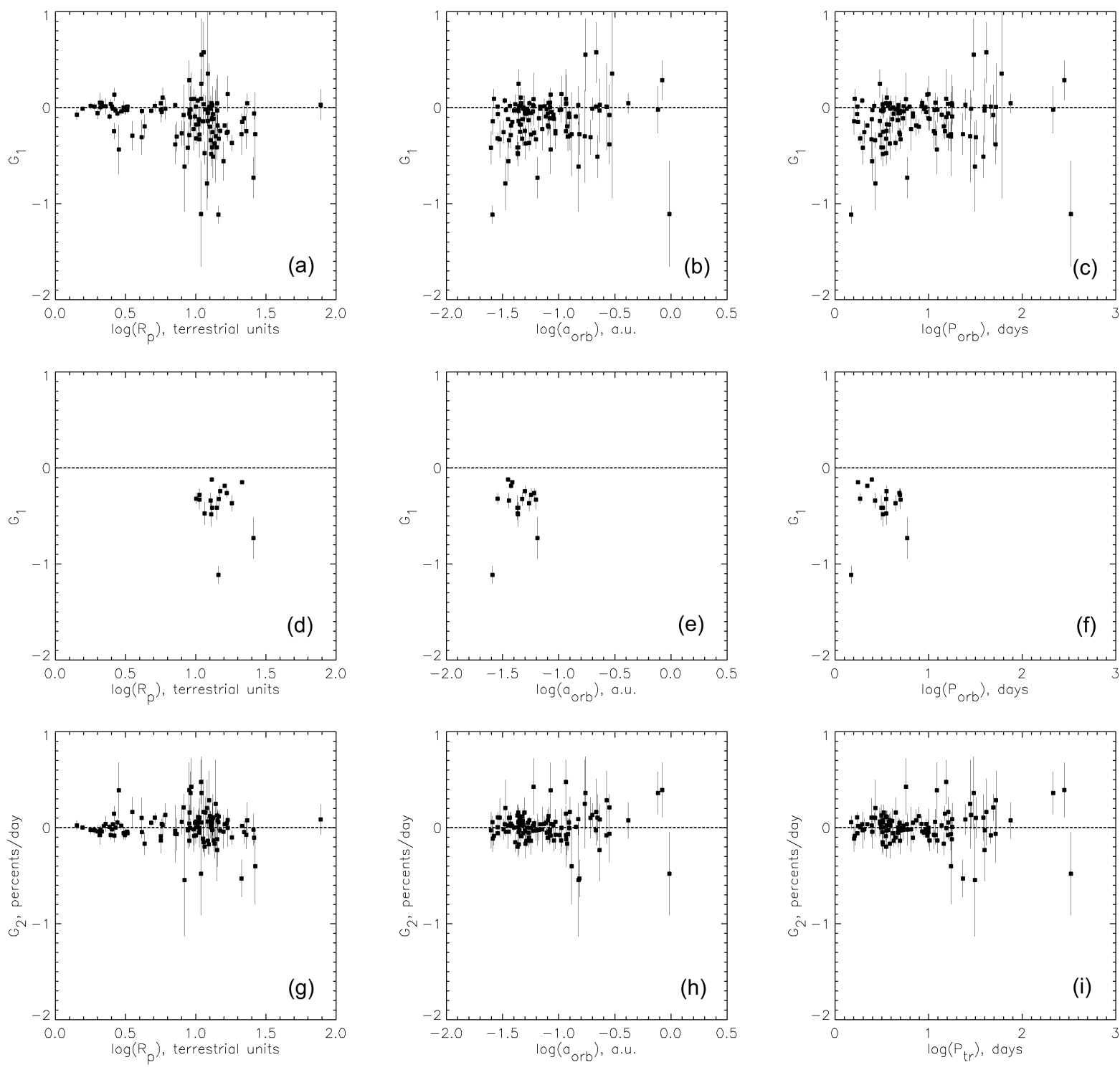

Fig. 5. Gradients $G_{1}$ and $G_{2}$, measured in the adjoining regions, versus planetary parameters: radius $R_{\mathrm{p}}$ of planet in $(a)$, $(d)$, and $(g)$; radius $a_{\mathrm{orb}}$ of orbit in $(b),(e)$, and $(h)$; and the orbital period $P_{\text {tr }}$ in $(c),(f)$, and $(i)$. The plots $(a)-(c)$ and $(g)-(i)$ show all the studied objects (squares with error bars) from Table C.1, while the middle row panels $(d)-(f)$ present only significant (above three standard errors) deviations of $\left|G_{1}\right|$ from zero (listed in Table 1).

Several deviated estimates with $G_{1}>0$ and $G_{2}<0$ could be a manifestation of the forward-scattering by micron dust. Atmospheric aerosols could result in the increase of flux only by $\delta\left(\Delta F_{k}\right)=32.5 \mathrm{ppm}$ for $\sim 1 \mu \mathrm{m}$ particles (see Table 2 in García \& Cabrera 2018) on the phase-angle scale $20^{\circ}$ (Eq. (2) in DeVore et al. 2016), which corresponds to the timescale $\tau_{s} \sim$ $\left(20^{\circ} / 360^{\circ}\right) P_{\mathrm{tr}}$ for the transit period $P_{\mathrm{tr}}$. Taking the typical $P_{\mathrm{tr}} \sim$ 10 days, one can estimate $\tau_{s} \sim 0.6$ days and $\left|G_{1,2}\right| \sim \delta\left(\Delta F_{k}\right) / \tau_{s}<$ $0.005 \% /$ day. This atmospheric aerosol contribution appears negligible in comparison with the obtained gradients $\left|G_{1,2}\right|>0.05 \% /$ day, which may however be not sufficiently reliable. For example, the TLC of Kepler-26b orbiting around KIC 9757613 shows the transits with maximal scattering-like gradients: $G_{1}=0.105 \pm 0.040 \% /$ day and $G_{2}=-0.158 \pm 0.047 \% /$ day at 2.6 and 3.3 standard-error confidence level, respectively.

Another population of the deviating gradient estimates with $G_{1}<0$ could be a manifestation of the large $(\gg 1 \mu \mathrm{m})$ obscuring particles. However, the contribution of such negative gradient cases in the total $G_{1}$-distribution in Fig. $2 b$ is insignificant. This is confirmed by an insignificant skewness, $0.35 \pm 0.23$, of the $G_{1}$-distribution (see Appendix A).

In summary, one can conclude that insignificant gradients $G_{1,2}$ in the distant region correspond to the mainly dustless environments. This nondetection result can also be considered as a verification of the light curve processing method used, which does not generate any systematic errors or artifacts.

Closer to planets, that is, in the adjoining pre- and posttransit regions with $\tau_{\min }=0.01$ days and $\tau_{\max }=0.05$ days, the analogous $G_{2}$ versus $G_{1}$ diagram has a different appearance (Fig. 3). One can see the shift of many estimate points towards the negative $G_{1}$ (Fig. 3a,b) as well as the bimodal character of the $G_{1}$-histogram (Fig. 3c). There is also an undisturbed normal population of gradient estimates clustered around $G_{1}=0$ (Fig. 3c) and $G_{2}=0$ (Fig. 3d). Another population of gradient estimates with $G_{1}<0$ and $G_{2} \sim 0$ (see Fig. 3d) might be related with the pre-transit manifestations of DOM; its existence is also confirmed by the significant skewness $-0.96 \pm 0.23$ of the $G_{1}$-distribution in Fig. $3 \mathrm{c}$. 

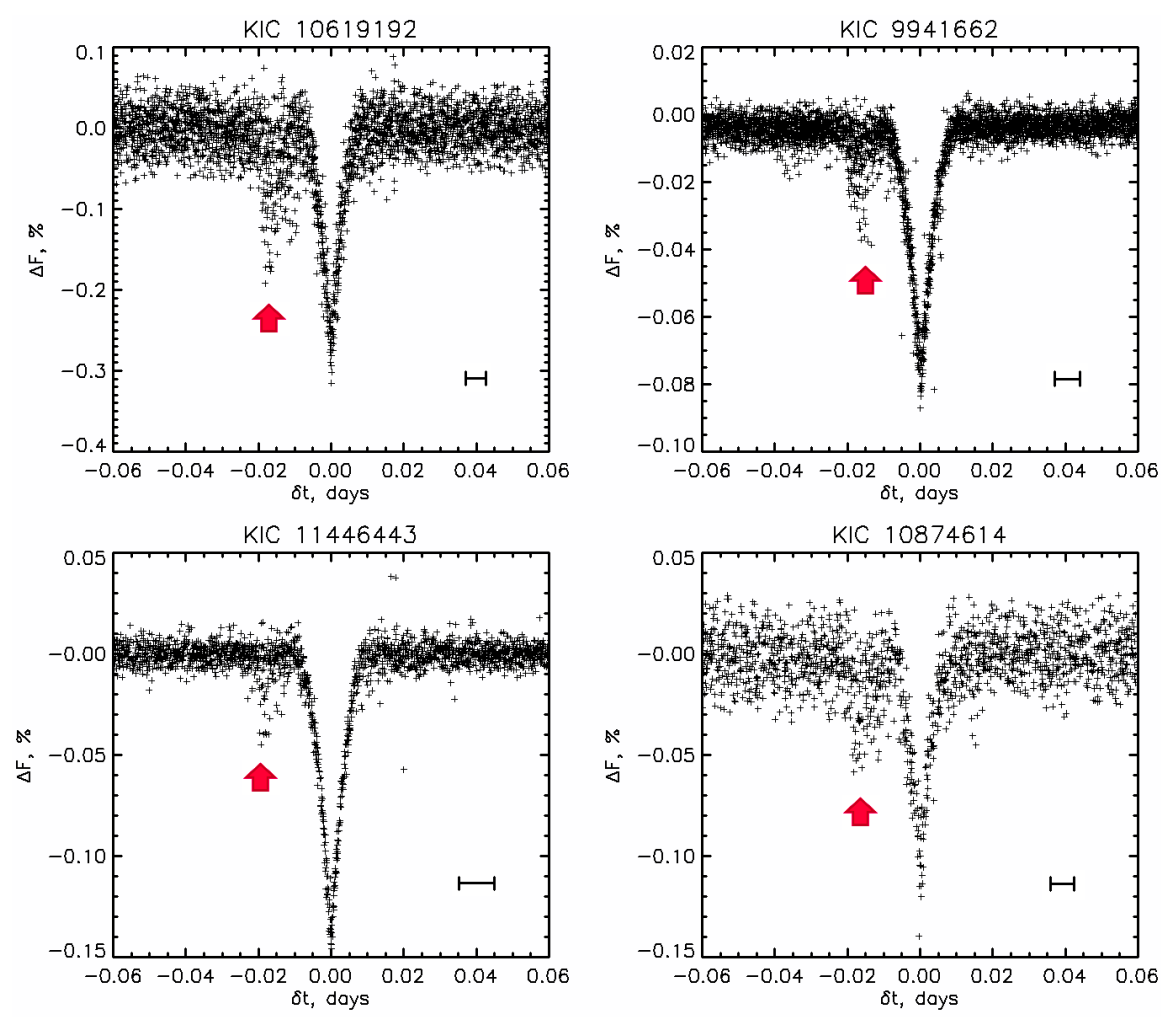

Fig. 6. Examples of DOM manifestations (arrowed) in the pre-ingress parts of folded and clipped TLCs (pluses), showing significant values of gradient $G_{1}$. The horizontal bars correspond to the radii of considered planets, i.e., ingress-time.

Table 1. Objects with a significant manifestation of pre-transit DOM.

\begin{tabular}{ccccccc}
\hline \hline KIC $^{(a)}$ & KOI $^{(b)}$ & $\begin{array}{c}R_{\mathrm{p}}{ }^{(c)} \\
\text { Earth's radii }\end{array}$ & $\begin{array}{c}a_{\text {orb }}{ }^{(c)} \\
\text { a.u. }\end{array}$ & $\begin{array}{c}P_{\text {tr }}{ }^{(c)} \\
\text { days }\end{array}$ & $\begin{array}{c}\underline{G}_{1}{ }^{(d)} \\
\sigma_{G_{1}}\end{array}$ & $\begin{array}{c}S_{\text {DOM }} \\
\times 10^{8} \mathrm{~km}^{2}\end{array}$ \\
\hline 5358624 & 830.01 & 11.58 & 0.043 & 3.53 & -4.1 & $2.27 \pm 0.56$ \\
5780885 & 97.01 & 16.66 & 0.061 & 4.89 & -5.6 & $7.26 \pm 1.30$ \\
6922244 & 10.01 & 14.59 & 0.047 & 3.52 & -4.7 & $5.21 \pm 1.11$ \\
7778437 & 131.01 & 10.63 & 0.063 & 5.01 & -3.3 & $4.29 \pm 1.31$ \\
8191672 & 18.01 & 14.92 & 0.050 & 3.55 & -4.4 & $5.61 \pm 1.29$ \\
9410930 & 196.01 & 10.05 & 0.029 & 1.86 & -5.6 & $2.18 \pm 0.39$ \\
9651668 & 183.01 & 12.73 & 0.036 & 2.68 & -4.2 & $2.36 \pm 0.56$ \\
9941662 & 13.01 & 21.42 & 0.039 & 1.76 & -12.0 & $10.32 \pm 0.86$ \\
10619192 & 203.01 & 14.48 & 0.026 & 1.49 & -12.0 & $8.79 \pm 0.73$ \\
10666592 & 2.01 & 16.10 & 0.038 & 2.20 & -8.7 & $5.43 \pm 0.63$ \\
10874614 & 17.01 & 13.06 & 0.044 & 3.23 & -7.6 & $5.27 \pm 0.69$ \\
10904857 & 194.01 & 14.16 & 0.043 & 3.12 & -3.3 & $3.13 \pm 0.96$ \\
11359879 & 128.01 & 10.62 & 0.057 & 4.94 & -4.1 & $2.04 \pm 0.50$ \\
11404644 & 6085.01 & 25.80 & 0.065 & 5.90 & -3.4 & $6.66 \pm 1.95$ \\
11446443 & 1.01 & 13.04 & 0.035 & 2.47 & -4.8 & $0.85 \pm 0.18$ \\
11804465 & 20.01 & 18.05 & 0.055 & 4.44 & -4.5 & $5.62 \pm 1.26$ \\
12019440 & 186.01 & 12.91 & 0.043 & 3.24 & -3.8 & $3.77 \pm 0.99$ \\
\hline
\end{tabular}

Notes. ${ }^{(a)}$ Identification number of the host star in the Kepler Input Catalog (Kepler Mission Team, 2009). ${ }^{(b)}$ KOI number in the NASA Exoplanet Archive. ${ }^{(c)}$ Data according the NASA Exoplanet Archive (https://exoplanetarchive.ipac.caltech.edu/cgi-bin/ TblView/nph-tblView?app=ExoTbls\&config=cumulative).

${ }^{(d)}$ Ratio of $G_{1}$ to its standard error $\sigma_{G_{1}}$ from Table C.1.

Theoretically, the known phenomenon of transit timing variations (TTVs), that is, the time-shifts of a TLC as a whole, could blur the transit borders in the folded TLC and create misleading gradients, namely $G_{1}<0$ and $G_{2}>0$. However, such shifts are known to oscillate in time around the linearly predicted ephemeride time of the transit $t_{E}$ (Holczer et al. 2016). Correspondingly, the TTV effect should blur both ingress and egress parts of the folded TLC. Hence, TTV cannot explain the observed just pre-transit flux drops without a detectable post-transit effect.

Another factor that may affect a light curve is variation of the planetary phase (e.g., Esteves et al. 2015). However, variation of this kind gives negligible gradients $G_{\mathrm{ph}} \sim 10^{-4} A_{p} /\left(0.5 P_{\mathrm{tr}}\right)$, where $A_{p} \leq 150 \mathrm{ppm}$ is the planetary brightness amplitude (see Table 5-8 in Esteves et al. 2015), and the factor $10^{-4}$ is the transforming coefficient from ppm flux units into percent used here. Taking a typical transit period $P_{\mathrm{tr}} \sim 3$ days for hot Jupiters, one can estimate that $G_{\mathrm{ph}} \lesssim 0.01 \% /$ day. Therefore, the second peak at $G_{1} \approx 0.3 \% /$ day in Fig. $3 \mathrm{c}$ cannot be attributed to planetary phase variability.

An imperfect polynomial approximation of the transit reference level $F_{b}\left(t_{k}\right)$ might hypothetically produce a misleading DOM-like effect. However, since the polynomial approximation is made for the sufficiently long part of the light curve $\left( \pm 10 \Delta t_{\text {tr }}\right.$ around the mid of transit) without account of the transit itself (i.e., with the removed transit), the planetary circumference would not significantly affect this approximation. We simulated the effects of imperfect definition of $F_{b}\left(t_{k}\right)$ using its linear approximation instead of a sixth-order polynomial. The result was an increased dispersion of $\Delta F$ before and after the folded TLC without a noticeable systematic effect on $G_{1}$ or $G_{2}$ (see Fig. 4).

Figure 5d shows that significantly (above three standard errors) negative values of $G_{1}$ are clearly associated with the Jupiter-type planets with radii $10 \lesssim R_{\mathrm{p}} \lesssim 25$ (i.e., $1.0 \lesssim$ $\left.\log \left(R_{\mathrm{p}}\right) \lesssim 1.4\right)$ in units of the Earth radius. Since our data set includes the transits of objects sized up to $\approx 0.1$ Jovian radii, the appearance of a pronounced negative $G_{1}$ feature by the Jupitertype and larger objects is not a selection effect (compare Figs. 5a and d). According to Fig. 5e, the significantly negative gradients 

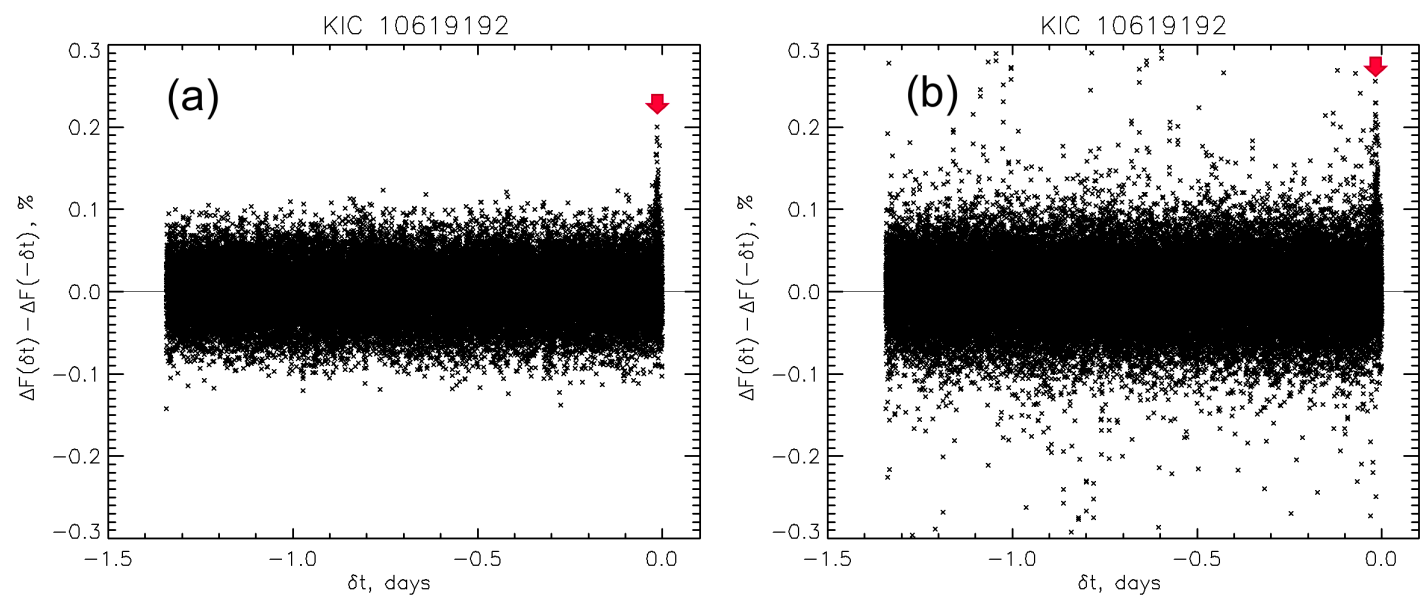

Fig. 7. Visualization of the pre-transit DOM manifestation (arrowed) in the light curve of KIC 10619192 above the background of cleared (panel a) and noncleared (panel $b$ ) folded out-of-transit parts $\left(P_{\mathrm{tr}}-\Delta t_{\mathrm{tr}}\right)$ of the light curve, i.e., during the whole transit period except for the transit itself.
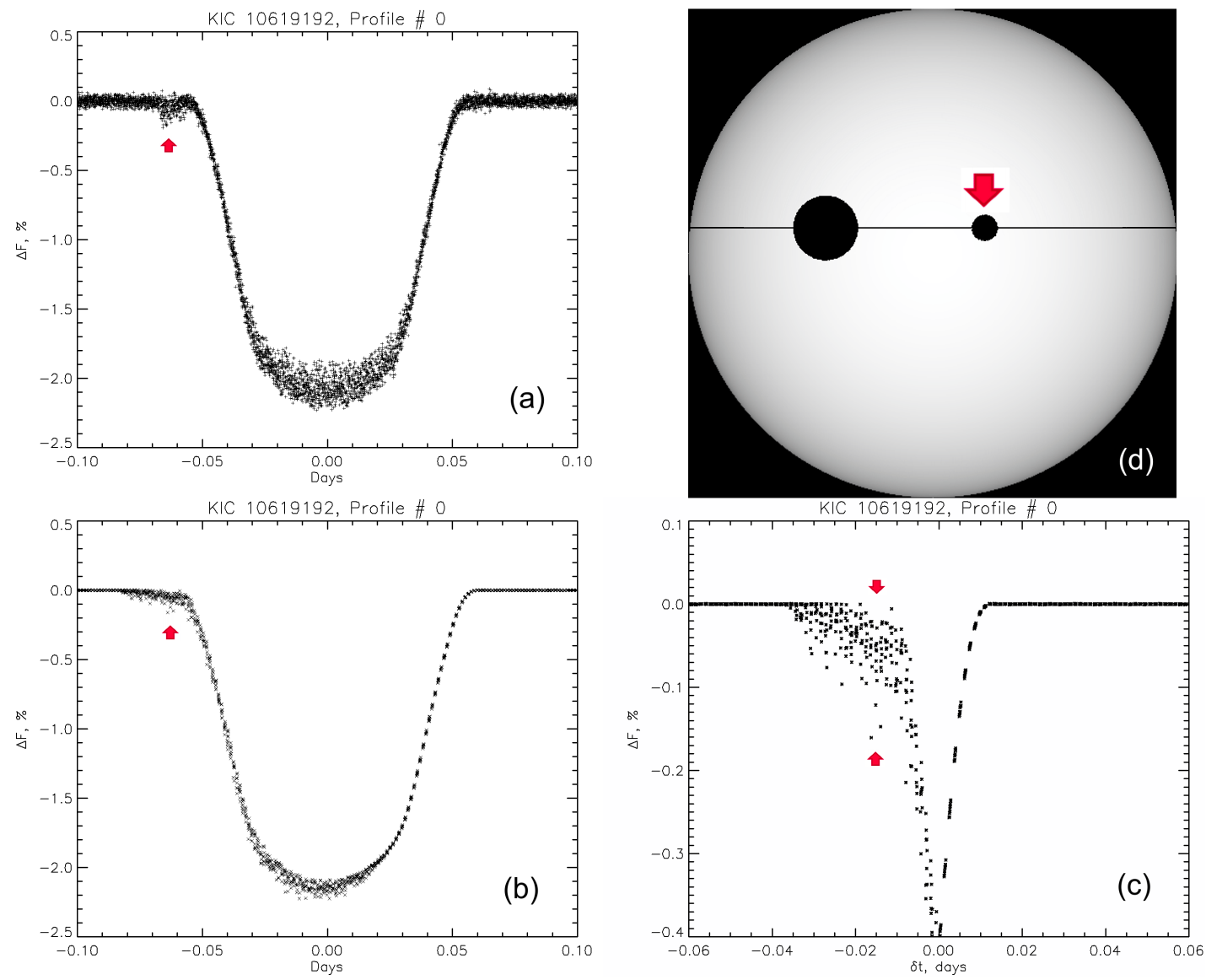

Fig. 8. Modeling of the pre-transit DOM manifestations (arrowed) in the case of KIC 10619192. Panel $a$ : real folded TLC; panel $b$ : synthetic folded TLC; panel $c$ : clipped TLC to visualize the synthetic DOM effect like in Fig. 5; panel d: model geometry with the real planet, crossing the stellar disk along the solid line together with an idealized DOM precursor (arrowed).

$G_{1}$ were found exclusively by hot Jupiters at extremely short orbits with radii $0.026 \lesssim a_{\text {orb }} \lesssim 0.065$ au, although the analyzed sample includes a wide range of objects with orbital distances of up to $1 \mathrm{au}$. A summary of the individual parameters of $17 \mathrm{KOIs}$, showing the most significant values of $G_{1}$, is given in Table 1 . This may indicate that the large size and short orbital distance of a planet are prerequisites of detectable DOM signatures. Moreover, the intensive material escape and mass-loss typical for the close-orbit hot jupiters might affect the evolution and distribution of the obscuring DOM. We note that no significant (beyond three standard errors) deviations of $\left|G_{2}\right|$ from zero were found among the considered objects (Figs. $5 \mathrm{~g}-\mathrm{i}$ ), meaning the absence of any measurement and/or calculation artifacts in the performed analysis. Therefore, the negative deviations of $G_{1}$ detected in the adjoining regions of a significant group of the studied objects appear to be real phenomena. 

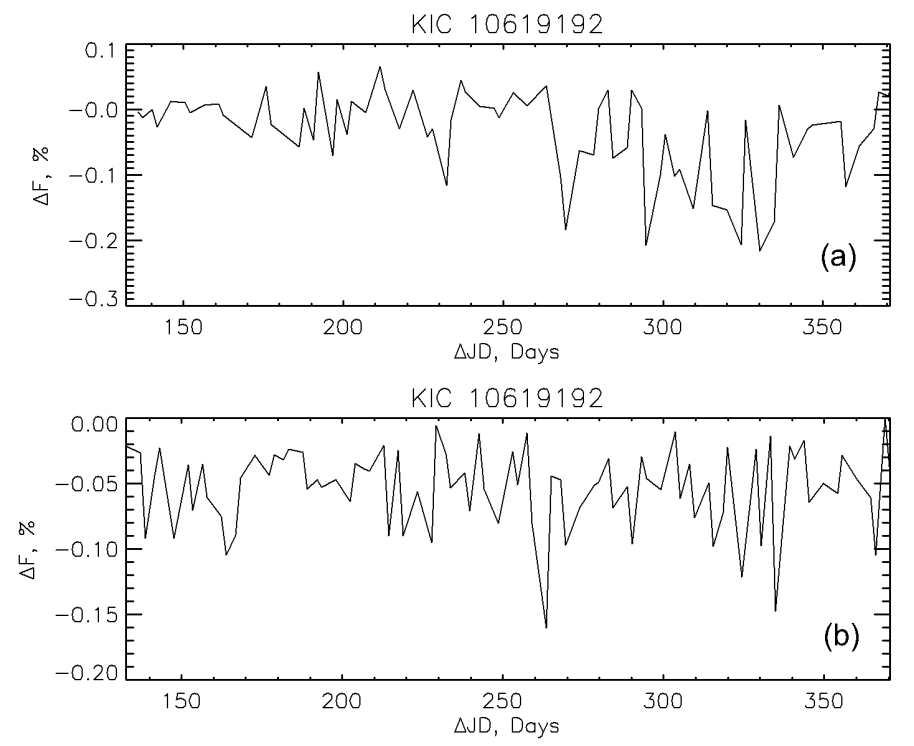

Fig. 9. Temporal variability of DOM effect as a pre-transit flux drop, measured at $-0.02<\delta t<-0.01$ days. Panel a: extraction from the real light curve of KIC 10619192; panel $b$ : analogous plot for synthetic light curve based on the used model. The abscissa scale is in differential barycentric Julian days $\Delta \mathrm{JD}$, counted from the reference day 2454833.0.

In Table 1 we also present estimations of the effective area obscured by DOM on the stellar disk as $S_{\text {DOM }} \approx$ $-0.01 \pi G_{1} \tau_{\max } R_{*}^{2}$ for the case of $G_{1}$ defined in the adjoining pretransit region. Here the coefficient 0.01 adopts the gradient, $G_{1}$, values, expressed in units of percent/day; the minus reflects the increase of the DOM particle concentration towards the planet; $\tau_{\max }=0.05$ days; and $R_{*}$ is the stellar radius from the NASA Exoplanet Archive. The estimated effective DOM area expressed in percent of the planetary cross-section varies in the considered set of objects from the minimal value of $0.4 \%$ achieved for KOI 1.01, up to the maximal value of $3.3 \%$ for KOI 203.01 .

The average value $\left\langle S_{\mathrm{DOM}}\right\rangle=4.8 \times 10^{8} \mathrm{~km}^{2}$ corresponds to the dust cloud of $N \approx S_{\text {DOM }} /(Q A) \sim 2.8 \times 10^{24}$ grains with the typical diameter $d \sim 10 \mu \mathrm{m}$ (like in the Solar System zodiacal cloud) and the geometrical cross-section $A=\pi(d / 2)^{2}$. Here, $Q=2.19$ is the extinction efficiency, that is, the ratio of the shadow cross-section of the particle and its geometrical crosssection $A$, which is calculated for a transparent sphere with the refractive index of 1.5 and the average wavelength of the Kepler photometry $\lambda=0.66 \mu \mathrm{m}$ using the Mie scattering theory ${ }^{2}$.

The integral volume of all these dust grains is $V_{d}=$ $(4 / 3) \pi(d / 2)^{3} N \sim 1.5 \mathrm{~km}^{3}$. It is in fact very small, as compared to the scales of potential dust sources; for example the Solar-System-like zodiacal cloud with an integral volume of its dust particles $\sim 1.4 \times 10^{4} \mathrm{~km}^{3}$, which is equivalent to a $15 \mathrm{~km}$-sized asteroid (Mennesson et al. 2019); a Jupiter-type KOI $\left(\sim 1.4 \times 10^{15} \mathrm{~km}^{3}\right)$ or its Io-like moon $\left(\sim 2.5 \times 10^{10} \mathrm{~km}^{3}\right)$. Altogether, the amount of dust estimated above, needed for the detected DOM manifestations, seems to be achievable with such orders-of-magnitude-larger sources.

Figure 6 shows the examples of the DOM-related features visible as an occasional decrease (arrowed) of radiation flux $\Delta F$ in the folded TLCs clustered in a narrow $(\approx 0.01$ day) range of pre-ingress time $\delta t$. There is a tendency for such features to repeat with the flux counting period 0.02 days (the best pronounced is the case of KIC 9941662). Apparently, this is a result

2 https://omlc.org/calc/mie_calc.html of artificial clustering of flux measurements at mid-exposure times.

The most manifested DOM-related feature in the TLC of KIC 10619192 (Fig. 6a) was studied in detail as a typical case. Figure 7 shows the difference between the pre-ingress and postegress parts of the folded TLC. One can see that the arrowed DOM-related feature is unique over the whole transit period. This means that this effect is really associated with the transiting object and its co-moving DOM structure, and is therefore unlikely to be an artifact.

We tried to simulate the DOM phenomenology assuming a hypothetic obscuring precursor (Fig. 8d) before the real planet (see also Appendix B). This precursor imitates a dust cloud ahead of the planet at a distance of five planetary radii. Since the same flux drop could be obtained with very different geometries and transparency of dust cloud, the equivalent opaque circular disk of the precursor was assumed for simplicity in the simulation.

Using the pixel-by-pixel integration over the stellar disk as in Fig. 8d, we achieved a certain resemblance between the modeled and real phase-folded TLCs (Figs. 8a,b) including the clustering effect (Fig. 8c). It is worth noting that the model parameters used in this simulation were neither fitted nor optimized to obtain an evident DOM-type effect. Only a rough empirical selection of parameters was done to find an appropriate scenario. An interesting feature consists in the fact that the synthetic TLC with the most similar behavior to observations was obtained in the simulations with a precursor that is stochastic in character. Specifically, the radius of the precursor was randomly taken in the range between zero and $0.5 R_{\mathrm{p}}$, whereas its appearance time was randomized in the range [0; 375 days] with an average time interval of 0.0375 days between individual DOM events. The duration of each DOM event was 0.02 days (i.e., the exposure time) with a constant area of eclipsing DOM for each individual event. The specific planetary and stellar parameters used in the simulation were taken for the object KIC 10619192 from the NASA Exoplanet Archive. Altogether, the model realistically reproduces the stochastic variability of flux decrease within the pre-transit time interval $-0.02<\delta t<-0.01$ days, related to the investigated DOM effect (see Fig. 9).

\section{Conclusions}

Below we summarize the major points, which may be concluded from the performed analysis and detections made.

1. The absence of systematic deviation of the out-of-transit gradients $G_{1}$ and $G_{2}$ from zero in the distant regions $(0.03<$ $|\delta t|<0.16$ days), and the same in the adjoining post-transit region $\left(0.01<\delta t<0.05\right.$ days), but only for $G_{2}$, means that there are no detectable DOM manifestations far from exoplanets, and in the closer regions behind them. This assures also the absence of influential artifacts from photometry or light curve processing.

2. Since the discovered significant photometrical peculiarities (irregular drops of stellar radiation flux) take place only before the ingress (Figs. 3 and 4) in close vicinity of the transit border (Figs. 5 and 6) of only short-period giant exoplanets, and this effect was not pointed out in previous studies, we deal here with a hitherto unknown exoplanetary phenomenon. It appears to be a new aspect of hot Jupiter nature requiring investigation.

3. The entirely pre-transit location of the found peculiarities excludes the possibility that these are photometric or processing artifacts (as explained in Sect. 3) or the manifestation of orbiting bodies (exomoons, moonlets, exo-rings, etc.) as a source of obscuring matter. 
4. The obtained results inspire the modeling work to simulate dusty atmospheric outflows (suggested in e.g., Wang \& Dai 2019), which interact with the stellar winds, being compressed in front of the material-losing exoplanets (like in Shaikhislamov et al. 2016; Dwivedi et al. 2019). An alternative scenario could involve the retarding of exozodiacal dust relative to the planet by the Poynting-Robertson effect leading to dust accumulation in an electrostatic, magnetic, or dynamic trap before the planet. These scenarios require a dedicated modeling which will be the subject of future work.

Acknowledgements. The authors acknowledge the projects I2939-N27 and S11606-N16 of the Austrian Science Fund (FWF) for the support. M.L.K. is grateful also for the grant No. 18-12-00080 of the Russian Science Foundation.

\section{References}

Aizawa, M., Masuda, K., Kawahara, H., \& Suto, Y. 2018, AJ, 155, 2018 Brogi, M., Keller, C. U., de Juan Ovelar, M., et al. 2012, A\&A, 545, L5 Budaj J. 2013, A\&A, 557, A72

Claret, A., \& Bloemen, S. 2011, A\&A, 529, A75
DeVore, J., Rappaport, S., Sanchis-Ojeda, R., Hoffman, K., \& Rowe, J. 2016, MNRAS, 461, 2453

Dwivedi, N. K., Khodachenko, M. L., Shaikhislamov, I. F., et al. 2019, MNRAS, 487, 4208

Esteves, L. J., De Mooij, E. J. W., \& Jayawardhana, R. 2015, ApJ, 804, 150

Garai, Z. 2018, A\&A, 611, A63

García, M. A., \& Cabrera, J. 2018, MNRAS, 473, 1801

Holczer, T., Mazeh, T., Nachmani, G., et al. 2016, ApJS, 225, 9

Huitson, C. M., Sing, D. K., Vidal-Madjar, A., et al. 2012, MNRAS, 422, 2477

Jenkins, J. M., Douglas, A. C., Chandrasekaran, H., et al. 2010, ApJ, 713, L87

Joanes, D. N., \& Gill, C. A. 1998, The Statistician, 47, 183

Juvan, I. G., Lendl, M., Cubillos, P. E., et al. 2018, A\&A, 610, A15

Kendall, M. G., \& Stuart, A. 1969, The Advanced Theory of Statistics (Houston: Griffin), 1

Lammer, H., \& Khodachenko, M. 2015, Characterizing Stellar and Exoplanetary Environments (Heidelberg: Springer), 2015

Mennesson, B., Kennedy, G., Ertel, S., et al. 2019, BASS, 51, 324

Sanchis-Ojeda, R., Rappaport, S., Pallè, E., et al. 2015, ApJ, 812, 112

Shaikhislamov, I. F., Khodachenko, M. L., Lammer, H., et al. 2016, ApJ, 832, 173

Shaikhislamov, I. F., Khodachenko, M. L., Lammer, H., et al. 2018, MNRAS, 481,5315

Spahn, F., Sachse, M., Seiß, M., et al. 2019, Space Sci. Rev., 215, 11

Teachey, A., Kipping, D. M., \& Schmitt, A. R. 2018, AJ, 155, 36

Wang, L., \& Dai, F. 2019, ApJ, 873, L1 


\section{Appendix A: Skewness of $G_{1,2}$ distributions}

To characterize the asymmetry of a distribution, we calculated the sample skewness (Joanes \& Gill 1998) of the obtained estimates of gradients $G_{1,2}$

$S_{1,2}=\frac{\sqrt{n(n-1)}}{n-2}\left\{\frac{\frac{1}{n} \sum_{i=1}^{n}\left(G_{1,2}^{i}-\left\langle G_{1,2}\right\rangle\right)^{3}}{\left[\frac{1}{n} \sum_{i=1}^{n}\left(G_{1,2}^{i}-\left\langle G_{1,2}\right\rangle\right)^{2}\right]^{3 / 2}}\right\}$,

where: $G_{1,2}^{i}$ is the estimate of $G_{1,2}$ for an individual KOI with a number $i ; n$ is the total number of considered objects; $\left\langle G_{1,2}\right\rangle$ is the average value over all estimates $G_{1,2}^{i}$. For the normal distribution the sample skewness has an expected value $S_{1,2}=0$ and the variance $\sigma_{G}^{2}=6 n(n-1) /[(n-2)(n+1)(n+3)]$ (Kendall \& Stuart 1969).

\section{Appendix B: Modeling of DOM effect in a TLC}

Since various shapes and transparencies of a transiting dust cloud are possible, a universal method of pixel-by-pixel integration (Juvan et al. 2018) suitable for any shape of transiting object is applied to compute the corresponding TLCs. The dimming of stellar flux during transit is characterized by the part of starlight blocked by the transiting object

$\Delta F=\frac{\iint I(x, y) \mathrm{d} x \mathrm{~d} y}{\iint I_{S}(x, y) \mathrm{d} x \mathrm{~d} y}$,

where the coordinate system co-centered with the stellar disk with $x$-axis parallel to the planet orbit projection on the stellar disk is used; $I_{s}$ is radiation intensity at a given position $(x, y)$ on the visible stellar disk, and $I$ is the same intensity but disturbed by the transiter. The above-mentioned integrals can be replaced by sums over $N_{p}$ pixels with serial number $i$ in the identical sets:
$\Delta F=\frac{\sum_{i=1}^{N_{p}} I\left(x_{i}, y_{i}\right)}{\sum_{i=1}^{N_{p}} I_{s}\left(x_{i}, y_{i}\right)}$.

The stellar limb darkening is taken into account according to the best (four coefficients) approximation by Claret \& Bloemen (2011), depending on particular stellar effective temperature and gravity, adopted from the NASA Exoplanet Archive. The planetary data (radius $R_{\mathrm{p}}$, semi-major axis $a_{\text {orb }}$ of the orbit, impact parameter $\beta$, mid-time $t_{0}$ of the first observed transit, transit period $P_{\mathrm{tr}}$ ) are also taken from the NASA Exoplanet Archive.

In the used reference system, the center exoplanetary shadow has coordinates

$x_{p}=a_{\mathrm{orb}} \sin \left[\frac{2 \pi}{P_{\mathrm{tr}}}\left(t_{k}-t_{0}\right)\right]$,

$y_{p}=\beta R_{\mathrm{s}}$,

where $R_{\mathrm{S}}$ is the stellar radius from the NASA Exoplanet Archive. The DOM is imitated by an opaque equivalent disk (see explanation in Sect. 3) at $x_{\mathrm{DOM}}=x_{p}+d$ and $y_{\mathrm{DOM}}=y_{p}$. Here parameter $d=5 R_{\mathrm{p}}$ is a constant effective distance selected empirically but without a detailed fitting. The time step for $\Delta F$ calculation is adopted as $\tau_{s}=0.00204$ days. The next procedure consists in the smoothing of the obtained synthetic light curve with time step $10 \tau_{s}$ which reproduces the real effect of the long-cadence integration time 0.0204 days. Consequently, the smoothing reproduces a certain widening of the long-cadence TLC, manifested as sharp out-of-transit minima (synthesized in Fig. 8c) at $0<|\delta t|<0.01$ days as seen in Figs. 1c and 6 for real TLCs. Moreover, the association of flux counts with the medial times of long-cadence exposures generates the 0.02-days periodicity in TLC, as for example in the case of KIC 9941662 in Fig. 6. We note that the synthetic light curves are treated further with the same processing pipeline as the real light curves. 


\section{Appendix C: Supplementary material}

Table C.1. Analyzed target set and processing results.

\begin{tabular}{|c|c|c|c|c|c|}
\hline \multirow{2}{*}{$\begin{array}{l}\mathrm{KIC}^{(a)} \\
\text { number }\end{array}$} & \multirow{2}{*}{$\begin{array}{l}\text { KOI }^{(b)} \\
\text { number }\end{array}$} & \multicolumn{2}{|c|}{ Adjoining region } & \multicolumn{2}{|c|}{ Distant region } \\
\hline & & $\begin{array}{c}G_{1} \\
\% \text { day }^{-1}\end{array}$ & $\begin{array}{c}G_{2} \\
\% \text { day }^{-1}\end{array}$ & $\begin{array}{c}G_{1} \\
\% \text { day }^{-1}\end{array}$ & $\begin{array}{c}G_{2} \\
\% \text { day }^{-1}\end{array}$ \\
\hline 2444412 & 103.01 & $-0.244 \pm 0.083$ & $0.146 \pm 0.079$ & $0.012 \pm 0.011$ & $-0.002 \pm 0.012$ \\
\hline 2571238 & 84.01 & $0.003 \pm 0.037$ & $-0.019 \pm 0.036$ & $-0.001 \pm 0.006$ & $-0.019 \pm 0.006$ \\
\hline 2581316 & 3681.01 & - & - & $0.006 \pm 0.038$ & $0.002 \pm 0.022$ \\
\hline 2987027 & 197.01 & $-0.300 \pm 0.153$ & $-0.068 \pm 0.165$ & $0.054 \pm 0.025$ & $0.022 \pm 0.026$ \\
\hline 3351888 & 801.01 & $0.093 \pm 0.099$ & $-0.086 \pm 0.097$ & $-0.008 \pm 0.016$ & $-0.008 \pm 0.017$ \\
\hline 3544595 & 69.01 & $-0.012 \pm 0.011$ & $0.001 \pm 0.010$ & $0.002 \pm 0.001$ & $-0.001 \pm 0.001$ \\
\hline 3545478 & 366.01 & $0.046 \pm 0.101$ & $0.075 \pm 0.188$ & $0.022 \pm 0.025$ & $-0.025 \pm 0.027$ \\
\hline 3749365 & 1176.01 & $-0.418 \pm 0.172$ & $-0.025 \pm 0.190$ & $-0.013 \pm 0.030$ & $0.056 \pm 0.031$ \\
\hline 3762468 & 208.01 & $0.249 \pm 0.145$ & $0.056 \pm 0.149$ & $-0.053 \pm 0.033$ & $0.091 \pm 0.034$ \\
\hline 3835670 & 149.01 & $-0.194 \pm 0.121$ & $-0.166 \pm 0.117$ & $0.024 \pm 0.020$ & $0.014 \pm 0.019$ \\
\hline 3847907 & 229.01 & $0.105 \pm 0.104$ & $0.026 \pm 0.107$ & $-0.013 \pm 0.017$ & $-0.016 \pm 0.018$ \\
\hline 3861595 & 4.01 & $0.044 \pm 0.034$ & $0.075 \pm 0.037$ & $-0.017 \pm 0.008$ & $-0.000 \pm 0.008$ \\
\hline 3935914 & 809.01 & $-0.142 \pm 0.137$ & $-0.114 \pm 0.134$ & $-0.030 \pm 0.022$ & $-0.020 \pm 0.022$ \\
\hline 4055765 & 100.01 & $0.142 \pm 0.184$ & $-0.001 \pm 0.196$ & $-0.007 \pm 0.028$ & $0.001 \pm 0.030$ \\
\hline 4150804 & 3678.01 & - & - & $-0.035 \pm 0.044$ & $0.010 \pm 0.053$ \\
\hline 4178389 & 185.01 & $-0.276 \pm 0.214$ & $-0.528 \pm 0.193$ & $0.005 \pm 0.036$ & $0.039 \pm 0.036$ \\
\hline 4180280 & 144.01 & $0.000 \pm 0.065$ & $-0.056 \pm 0.065$ & $0.010 \pm 0.011$ & $0.019 \pm 0.011$ \\
\hline 4349452 & 244.01 & $0.009 \pm 0.023$ & $-0.018 \pm 0.021$ & $0.002 \pm 0.003$ & $-0.003 \pm 0.003$ \\
\hline 4742414 & 631.01 & $-0.096 \pm 0.098$ & $-0.005 \pm 0.098$ & $-0.035 \pm 0.015$ & $-0.005 \pm 0.015$ \\
\hline 4833421 & 232.01 & $-0.020 \pm 0.132$ & $-0.133 \pm 0.133$ & $-0.023 \pm 0.021$ & $0.019 \pm 0.023$ \\
\hline 5084942 & 161.01 & $-0.030 \pm 0.037$ & $0.002 \pm 0.038$ & $0.009 \pm 0.006$ & $-0.000 \pm 0.007$ \\
\hline 5357901 & 188.01 & $-0.177 \pm 0.090$ & $0.063 \pm 0.081$ & $0.033 \pm 0.014$ & $-0.009 \pm 0.014$ \\
\hline 5358624 & 830.01 & $-0.473 \pm 0.117$ & $-0.198 \pm 0.105$ & $-0.032 \pm 0.017$ & $-0.013 \pm 0.017$ \\
\hline 5383248 & 261.01 & $-0.059 \pm 0.031$ & $0.053 \pm 0.033$ & $-0.002 \pm 0.006$ & $-0.008 \pm 0.006$ \\
\hline 5651104 & 840.01 & $-0.287 \pm 0.134$ & $0.042 \pm 0.124$ & $0.027 \pm 0.022$ & $0.018 \pm 0.022$ \\
\hline 5728139 & 206.01 & $-0.112 \pm 0.115$ & $-0.023 \pm 0.106$ & $-0.022 \pm 0.022$ & $0.039 \pm 0.022$ \\
\hline 5735762 & 148.02 & $0.136 \pm 0.068$ & $-0.082 \pm 0.069$ & $0.006 \pm 0.012$ & $-0.007 \pm 0.011$ \\
\hline 5780885 & 97.01 & $-0.261 \pm 0.046$ & $0.045 \pm 0.045$ & $-0.004 \pm 0.006$ & $0.011 \pm 0.006$ \\
\hline 5794240 & 254.01 & $-0.329 \pm 0.193$ & $0.105 \pm 0.201$ & $0.039 \pm 0.033$ & $0.013 \pm 0.033$ \\
\hline 5812701 & 12.01 & $0.025 \pm 0.196$ & $0.088 \pm 0.168$ & $-0.028 \pm 0.038$ & $-0.005 \pm 0.037$ \\
\hline 5866724 & 85.01 & $-0.012 \pm 0.021$ & $0.036 \pm 0.021$ & $0.003 \pm 0.003$ & $-0.001 \pm 0.003$ \\
\hline 5972334 & 191.01 & $0.093 \pm 0.308$ & $0.475 \pm 0.230$ & $0.029 \pm 0.051$ & $-0.060 \pm 0.046$ \\
\hline 6029239 & 304.01 & $0.049 \pm 0.039$ & $-0.037 \pm 0.040$ & $-0.008 \pm 0.008$ & $-0.003 \pm 0.008$ \\
\hline 6046540 & 200.01 & $-0.184 \pm 0.108$ & $0.077 \pm 0.108$ & $-0.001 \pm 0.017$ & $0.004 \pm 0.017$ \\
\hline 6061119 & 846.01 & $-0.298 \pm 0.478$ & $0.247 \pm 0.459$ & $0.008 \pm 0.081$ & $0.008 \pm 0.078$ \\
\hline 6522242 & 855.01 & $0.575 \pm 0.315$ & $0.162 \pm 0.347$ & $0.090 \pm 0.059$ & $-0.048 \pm 0.061$ \\
\hline 6526710 & 856.01 & $-0.029 \pm 0.329$ & $-0.231 \pm 0.325$ & $0.121 \pm 0.075$ & $0.079 \pm 0.069$ \\
\hline 6541920 & 157.03 & $-0.309 \pm 0.181$ & $0.102 \pm 0.212$ & $0.025 \pm 0.029$ & $0.028 \pm 0.034$ \\
\hline 6634112 & 5308.01 & $-0.110 \pm 0.125$ & $-0.045 \pm 0.121$ & $0.005 \pm 0.021$ & $-0.011 \pm 0.019$ \\
\hline 6842345 & 6774.01 & $-0.275 \pm 0.436$ & $-0.402 \pm 0.395$ & $0.036 \pm 0.065$ & $0.023 \pm 0.070$ \\
\hline 6849046 & 201.01 & $-0.063 \pm 0.058$ & $-0.137 \pm 0.058$ & $-0.008 \pm 0.009$ & $0.022 \pm 0.009$ \\
\hline 6850504 & 70.01 & $0.018 \pm 0.036$ & $-0.024 \pm 0.033$ & $-0.013 \pm 0.005$ & $0.013 \pm 0.005$ \\
\hline 6922244 & 10.01 & $-0.324 \pm 0.068$ & $0.118 \pm 0.056$ & $-0.009 \pm 0.010$ & $0.010 \pm 0.009$ \\
\hline 7046804 & 205.01 & $-0.267 \pm 0.129$ & $0.062 \pm 0.132$ & $-0.025 \pm 0.022$ & $0.005 \pm 0.021$ \\
\hline 7051180 & 64.01 & $0.073 \pm 0.031$ & $0.035 \pm 0.032$ & $-0.005 \pm 0.005$ & $0.007 \pm 0.005$ \\
\hline 7380537 & 883.01 & $-0.787 \pm 0.276$ & $0.204 \pm 0.291$ & $0.012 \pm 0.043$ & $-0.084 \pm 0.045$ \\
\hline 7603200 & 314.01 & $-0.073 \pm 0.052$ & $0.024 \pm 0.050$ & $0.010 \pm 0.010$ & $0.016 \pm 0.010$ \\
\hline 7626506 & 150.02 & $0.013 \pm 0.079$ & $0.039 \pm 0.079$ & $0.019 \pm 0.013$ & $-0.005 \pm 0.013$ \\
\hline 7778437 & 131.01 & $-0.329 \pm 0.100$ & $-0.030 \pm 0.101$ & $-0.007 \pm 0.014$ & $0.018 \pm 0.016$ \\
\hline
\end{tabular}

Notes. ${ }^{(a)}$ Identification number of the host star in the Kepler Input Catalog (Kepler Mission Team, 2009). ${ }^{(b)}$ Number of the Kepler Object of Interest in the NASA Exoplanet Archive. 
Table C.1. continued.

\begin{tabular}{|c|c|c|c|c|c|}
\hline \multirow{2}{*}{$\begin{array}{l}\mathrm{KIC}^{(a)} \\
\text { number }\end{array}$} & \multirow{2}{*}{$\begin{array}{l}\text { KOI }^{(b)} \\
\text { number }\end{array}$} & \multicolumn{2}{|c|}{ Adjoining region } & \multicolumn{2}{|c|}{ Distant region } \\
\hline & & $\begin{array}{c}G_{1} \\
\% \text { day }^{-1}\end{array}$ & $\begin{array}{c}G_{2} \\
\% \text { day }^{-1}\end{array}$ & $\begin{array}{c}G_{1} \\
\% \text { day }^{-1}\end{array}$ & $\begin{array}{c}G_{2} \\
\% \text { day }^{-1}\end{array}$ \\
\hline 7849854 & 897.01 & $-0.256 \pm 0.118$ & $-0.016 \pm 0.109$ & $0.020 \pm 0.019$ & $-0.021 \pm 0.019$ \\
\hline 7877496 & 202.01 & $-0.065 \pm 0.052$ & $0.106 \pm 0.056$ & $-0.012 \pm 0.008$ & $0.004 \pm 0.008$ \\
\hline 7906882 & 686.01 & $0.009 \pm 0.450$ & $0.284 \pm 0.307$ & $0.013 \pm 0.088$ & $-0.024 \pm 0.060$ \\
\hline 7950644 & 192.01 & $-0.117 \pm 0.118$ & $-0.131 \pm 0.113$ & $0.004 \pm 0.018$ & $0.034 \pm 0.018$ \\
\hline 8191672 & 18.01 & $-0.241 \pm 0.055$ & $-0.028 \pm 0.047$ & $-0.022 \pm 0.008$ & $-0.002 \pm 0.007$ \\
\hline 8255887 & 908.01 & $-0.129 \pm 0.144$ & $-0.050 \pm 0.155$ & $-0.007 \pm 0.025$ & $0.022 \pm 0.026$ \\
\hline 8260218 & 1066.01 & $0.088 \pm 0.272$ & $0.427 \pm 0.294$ & $0.031 \pm 0.041$ & $-0.039 \pm 0.046$ \\
\hline 8349582 & 122.01 & $-0.022 \pm 0.048$ & $-0.076 \pm 0.050$ & $0.004 \pm 0.008$ & $0.016 \pm 0.009$ \\
\hline 8359498 & 127.01 & $-0.174 \pm 0.064$ & $0.109 \pm 0.067$ & $-0.013 \pm 0.010$ & $-0.001 \pm 0.010$ \\
\hline 8394721 & 152.01 & $-0.382 \pm 0.206$ & $-0.063 \pm 0.297$ & $-0.013 \pm 0.042$ & $0.074 \pm 0.052$ \\
\hline 8456679 & 102.01 & $0.011 \pm 0.022$ & $-0.057 \pm 0.022$ & $-0.000 \pm 0.003$ & $-0.000 \pm 0.003$ \\
\hline 8478994 & 245.01 & $0.010 \pm 0.036$ & $-0.026 \pm 0.038$ & $-0.003 \pm 0.005$ & $0.008 \pm 0.005$ \\
\hline 8544996 & 913.01 & $-0.118 \pm 0.139$ & $0.070 \pm 0.130$ & $-0.004 \pm 0.021$ & $-0.017 \pm 0.023$ \\
\hline 8554498 & 5.01 & $0.025 \pm 0.021$ & $-0.034 \pm 0.021$ & $0.003 \pm 0.003$ & $0.005 \pm 0.003$ \\
\hline 8644288 & 137.02 & - & - & $-0.035 \pm 0.017$ & $0.007 \pm 0.017$ \\
\hline 8684730 & 319.01 & $0.012 \pm 0.123$ & $-0.078 \pm 0.086$ & $-0.014 \pm 0.019$ & $-0.005 \pm 0.018$ \\
\hline 8711794 & 105.01 & $-0.015 \pm 0.049$ & $-0.050 \pm 0.048$ & $0.000 \pm 0.008$ & $-0.006 \pm 0.008$ \\
\hline 8866102 & 42.01 & $-0.092 \pm 0.033$ & $0.007 \pm 0.029$ & $0.002 \pm 0.004$ & $0.000 \pm 0.005$ \\
\hline 8890783 & 464.01 & - & - & $0.135 \pm 0.098$ & $0.214 \pm 0.107$ \\
\hline 9115800 & 421.01 & $-0.017 \pm 0.141$ & $0.157 \pm 0.159$ & $0.000 \pm 0.025$ & $-0.017 \pm 0.025$ \\
\hline 9141746 & 929.01 & $-0.222 \pm 0.220$ & $-0.017 \pm 0.232$ & $0.044 \pm 0.036$ & $-0.015 \pm 0.038$ \\
\hline 9166862 & 931.01 & $-0.374 \pm 0.161$ & $-0.167 \pm 0.151$ & $0.008 \pm 0.026$ & $0.024 \pm 0.026$ \\
\hline 9305831 & 204.01 & $-0.007 \pm 0.108$ & $-0.172 \pm 0.106$ & $-0.018 \pm 0.017$ & $-0.021 \pm 0.017$ \\
\hline 9410930 & 196.01 & $-0.319 \pm 0.056$ & $0.004 \pm 0.052$ & $-0.009 \pm 0.008$ & $-0.010 \pm 0.008$ \\
\hline 9471974 & 119.01 & $-0.077 \pm 0.313$ & $0.209 \pm 0.139$ & $0.044 \pm 0.033$ & $-0.032 \pm 0.021$ \\
\hline 9579641 & 115.01 & $-0.040 \pm 0.039$ & $0.020 \pm 0.039$ & $-0.004 \pm 0.006$ & $-0.006 \pm 0.006$ \\
\hline 9595827 & 217.01 & $-0.009 \pm 0.134$ & $0.029 \pm 0.134$ & $-0.032 \pm 0.022$ & $-0.002 \pm 0.022$ \\
\hline 9631995 & 22.01 & $-0.199 \pm 0.073$ & $0.120 \pm 0.094$ & $0.012 \pm 0.011$ & $0.025 \pm 0.013$ \\
\hline 9651668 & 183.01 & $-0.338 \pm 0.079$ & $0.101 \pm 0.069$ & $-0.019 \pm 0.011$ & $-0.002 \pm 0.011$ \\
\hline 9757613 & 250.01 & $-0.435 \pm 0.255$ & $0.388 \pm 0.289$ & $0.105 \pm 0.040$ & $-0.158 \pm 0.047$ \\
\hline 9772531 & 950.01 & $-0.615 \pm 0.467$ & $-0.545 \pm 0.585$ & $-0.091 \pm 0.083$ & $0.048 \pm 0.089$ \\
\hline 9818381 & 135.01 & $-0.138 \pm 0.055$ & $0.063 \pm 0.052$ & $-0.002 \pm 0.009$ & $0.001 \pm 0.008$ \\
\hline 9941662 & 13.01 & $-0.147 \pm 0.012$ & $0.018 \pm 0.007$ & $-0.003 \pm 0.001$ & $-0.001 \pm 0.000$ \\
\hline 9962595 & 5745.01 & $-0.245 \pm 0.182$ & $-0.072 \pm 0.150$ & $0.012 \pm 0.027$ & $0.005 \pm 0.026$ \\
\hline 10019708 & 199.01 & $-0.251 \pm 0.096$ & $0.080 \pm 0.095$ & $-0.019 \pm 0.016$ & $-0.008 \pm 0.015$ \\
\hline 10187017 & 82.01 & $0.039 \pm 0.048$ & $-0.075 \pm 0.045$ & $0.011 \pm 0.008$ & $0.006 \pm 0.008$ \\
\hline 10264660 & 98.01 & $-0.092 \pm 0.036$ & $0.051 \pm 0.032$ & $-0.000 \pm 0.005$ & $-0.004 \pm 0.005$ \\
\hline 10295951 & 1154.01 & $-0.061 \pm 0.060$ & $-0.103 \pm 0.054$ & $-0.020 \pm 0.009$ & $0.015 \pm 0.008$ \\
\hline 10318874 & 104.01 & $-0.024 \pm 0.030$ & $-0.042 \pm 0.027$ & $0.007 \pm 0.005$ & $0.012 \pm 0.004$ \\
\hline 10619192 & 203.01 & $-1.113 \pm 0.092$ & $0.058 \pm 0.064$ & $-0.007 \pm 0.011$ & $0.007 \pm 0.011$ \\
\hline 10666592 & 2.01 & $-0.186 \pm 0.021$ & $0.035 \pm 0.016$ & $-0.010 \pm 0.002$ & $0.005 \pm 0.002$ \\
\hline 10748390 & 3.01 & $-0.033 \pm 0.025$ & $0.051 \pm 0.022$ & $0.000 \pm 0.003$ & $-0.003 \pm 0.003$ \\
\hline 10795103 & 3683.01 & $-0.020 \pm 0.246$ & $0.362 \pm 0.222$ & $-0.060 \pm 0.033$ & $-0.017 \pm 0.031$ \\
\hline 10874614 & 17.01 & $-0.414 \pm 0.054$ & $0.053 \pm 0.044$ & $-0.007 \pm 0.007$ & $-0.006 \pm 0.007$ \\
\hline 10904857 & 194.01 & $-0.413 \pm 0.126$ & $0.126 \pm 0.112$ & $0.007 \pm 0.019$ & $-0.032 \pm 0.019$ \\
\hline 10905239 & 46.01 & $0.045 \pm 0.051$ & $-0.087 \pm 0.055$ & $-0.012 \pm 0.008$ & $0.004 \pm 0.009$ \\
\hline 10925104 & 156.03 & $0.052 \pm 0.099$ & $-0.075 \pm 0.098$ & $-0.025 \pm 0.016$ & $-0.008 \pm 0.016$ \\
\hline 11046458 & 214.01 & $-0.022 \pm 0.078$ & $0.157 \pm 0.078$ & $0.012 \pm 0.013$ & $-0.022 \pm 0.014$ \\
\hline 11138155 & 760.01 & $-0.055 \pm 0.132$ & $-0.135 \pm 0.138$ & $0.010 \pm 0.024$ & $-0.032 \pm 0.023$ \\
\hline
\end{tabular}


Table C.1. continued.

\begin{tabular}{|c|c|c|c|c|c|}
\hline \multirow{2}{*}{$\begin{array}{l}\mathrm{KIC}^{(a)} \\
\text { number }\end{array}$} & \multirow{2}{*}{$\begin{array}{l}\text { KOI }^{(b)} \\
\text { number }\end{array}$} & \multicolumn{2}{|c|}{ Adjoining region } & \multicolumn{2}{|c|}{ Distant region } \\
\hline & & $\begin{array}{c}G_{1} \\
\% \text { day }^{-1}\end{array}$ & $\begin{array}{c}G_{2} \\
\% \text { day }^{-1}\end{array}$ & $\begin{array}{c}G_{1} \\
\% \text { day }^{-1}\end{array}$ & $\begin{array}{c}G_{2} \\
\% \text { day }^{-1}\end{array}$ \\
\hline 11295426 & 246.01 & $0.015 \pm 0.016$ & $0.017 \pm 0.017$ & $0.000 \pm 0.002$ & $-0.003 \pm 0.002$ \\
\hline 11359879 & 128.01 & $-0.279 \pm 0.068$ & $-0.002 \pm 0.066$ & $0.014 \pm 0.011$ & $-0.000 \pm 0.011$ \\
\hline 11391018 & 189.01 & $0.550 \pm 0.376$ & $0.361 \pm 0.378$ & $-0.030 \pm 0.073$ & $-0.011 \pm 0.083$ \\
\hline 11401755 & 277.01 & $-0.292 \pm 0.152$ & $0.163 \pm 0.154$ & $-0.092 \pm 0.015$ & $0.080 \pm 0.014$ \\
\hline 11404644 & 6085.01 & $-0.730 \pm 0.213$ & $-0.020 \pm 0.164$ & $-0.090 \pm 0.030$ & $-0.015 \pm 0.030$ \\
\hline 11442793 & 351.01 & $-1.106 \pm 0.549$ & $-0.478 \pm 0.432$ & $0.208 \pm 0.159$ & $0.102 \pm 0.139$ \\
\hline 11446443 & 1.01 & $-0.119 \pm 0.025$ & $0.023 \pm 0.021$ & $-0.003 \pm 0.002$ & $0.003 \pm 0.002$ \\
\hline 11449844 & 125.01 & $-0.511 \pm 0.219$ & $0.104 \pm 0.241$ & $0.097 \pm 0.037$ & $-0.027 \pm 0.040$ \\
\hline 11502867 & 195.01 & $0.035 \pm 0.102$ & $-0.151 \pm 0.104$ & $0.020 \pm 0.018$ & $0.023 \pm 0.017$ \\
\hline 11517719 & 1416.01 & $-0.558 \pm 0.201$ & $-0.043 \pm 0.173$ & $-0.028 \pm 0.027$ & $0.056 \pm 0.025$ \\
\hline 11554435 & 63.01 & $-0.046 \pm 0.052$ & $0.037 \pm 0.049$ & $0.013 \pm 0.008$ & $-0.007 \pm 0.009$ \\
\hline 11804465 & 20.01 & $-0.368 \pm 0.082$ & $-0.102 \pm 0.070$ & $0.012 \pm 0.011$ & $-0.000 \pm 0.010$ \\
\hline 11853905 & 7.01 & $-0.036 \pm 0.032$ & $-0.044 \pm 0.031$ & $-0.000 \pm 0.005$ & $0.006 \pm 0.005$ \\
\hline 11974540 & 129.01 & $0.030 \pm 0.158$ & $0.084 \pm 0.160$ & $-0.047 \pm 0.024$ & $0.045 \pm 0.025$ \\
\hline 12019440 & 186.01 & $-0.481 \pm 0.126$ & $0.005 \pm 0.113$ & $-0.023 \pm 0.019$ & $0.006 \pm 0.019$ \\
\hline 12105051 & 141.01 & $0.015 \pm 0.054$ & $0.104 \pm 0.052$ & $-0.011 \pm 0.009$ & $-0.009 \pm 0.008$ \\
\hline 12251650 & 621.01 & $0.046 \pm 0.294$ & $-0.121 \pm 0.283$ & $0.051 \pm 0.047$ & $-0.023 \pm 0.044$ \\
\hline 12252424 & 153.01 & $-0.056 \pm 0.062$ & $-0.039 \pm 0.063$ & $0.001 \pm 0.010$ & $0.009 \pm 0.010$ \\
\hline 12314973 & 279.01 & $-0.009 \pm 0.112$ & $0.131 \pm 0.121$ & $0.013 \pm 0.017$ & $0.007 \pm 0.024$ \\
\hline 12735740 & 3663.01 & $0.285 \pm 0.205$ & $0.393 \pm 0.284$ & $-0.080 \pm 0.051$ & $0.030 \pm 0.045$ \\
\hline
\end{tabular}

